\title{
Acknowledgment of Reviewers, 2019
}

The success of Phytopathology depends on the quality of manuscripts submitted by authors and on the care and competence with which they are reviewed. It is the policy of the Editorial Board to solicit reviews for manuscripts from specialists who are most qualified to review them. In addition to members of the Editorial Board, the individuals listed below provide constructive critical reviews of one or more manuscripts during the past year. Their names are published here in grateful appreciation of their contribution to the journal and to the science of phytopathology. https://dx.doi.org/10.1094/PHYTO-110-1-0004

Nilwala Abeysekara

James Adaskaveg

Scott Adkins

Catherine Aime

TS Ajayasree

Olutoyosi Ajayi

Eduard Akhunov

Olufemi Alabi

Erik Alexandersson

Sajid Ali

Caitilyn Allen

Adriana Alvarez

Reda Amezrou

Achour Amiri

Seanna Annis

Meriem Aoun

Tsutomu Arie

Andrew Armitage

Matthew Back

Ofir Bahar

Brian Bahder

Jinhe Bai

Natalia Bajsa

Guus Bakkeren

Marie-Helene Balesdent

David Baltrus

Maya Bar

Jayme Barbedo

Kendra Baumgartner

Fulya Baysal-Gurel

Franklin Behlau

Manuel Benedetti

Anna Berlin

Soraya Bertioli

Jan Bettgenhaeuser

David Bird

Kaitlyn Bissonnette

Melvin Bolton

Hossein Borhan

Richard Bostock

Robert Bowden

Helen Brabham

Gurcharn Singh Brar

Veronique Brault

Marcelo Brigido

Martin Broberg

Daren Brown

James Brown

Judith Brown

Greg Browne

May Brurberg

Alex Bucksch

Carolee Bull

Saul Burdman

Kathryn Bushley

Elliott Bussell

Lance Cadle-Davidson
Lei Cai

Angela Calderon

Fernando Casanoves

Pablo Castillo

Vittoria Catara

Gilles Cellier

Paulo Ceresini

Divya Chandran

Jeff Chang

Nikki Charlton

Jantasuriyarat Chatchawan

Subhadeep Chatterjee

Guoyue Chen

Jianchi Chen

Nicolas Chen

Qinghe Chen

Xiaoren Chen

Zhi-Yuan Chen

Martin Chilvers

Robin Choudhury

Zhaohui Chu

Sofia Chulze

Edwin Civerolo

Kelley Clark

Christopher Clarke

Gitta Coaker

Paul Cobine

Nicolas Cobo

Jeffery Coleman

Patrick Conner

David E. L. Cooke

Bret Cooper

Rodney Cooper

Bart Cottyn

John Couture

Christina Cowger

Kerik Cox

Jaime Cubero

Mladen Cucak

James Culver

Christian Cumagun

Nik Cunniffe

Johan Daeseger

John DaGraca

Louise-Marie Dandurand

Ciara Dangerfield

Amanda De La Torre

Luc de Lapeyre

Sophie de Vries

Ralph Dean

Thomas Debener

Emerson Del Ponte

Lisa DeVetter

Megan Dewdney

Shunping Ding

Leslie Domier

Ruairi Donnelly
Nicole Donofrio

Anne Dorrance

Peter Dracatos

Milton Drott

Lindsey du Toit

Shuo Duan

Zoe Dubrow

Jerome Duclercq

Nicholas Dufault

Jeremiah Dung

Bhabesh Dutta

Alan Dyer

Paul Esker

Katherine Evans

Tom Evans

Helen Eyles

Bryce Falk

Richard Falloon

Mark Farman

Travis Faske

Hao Feng

Jessie Fernandez

Julie Flood

Tom Forge

Jake Fountain

Bart Fraaije

Juliana Freitas-Astua

Timothy Friesen

Karyn Froud

William Fry

Zhen Fu

Dean Gabriel

Beata Gabrys

Xiquan Gao

Hernan Garcia-Ruiz

Weyessa Garedew

Adrien Gauthier

David Gent

Hugo Germain

Murad Ghanim

Godelieve Gheysen

Pierre Gladieux

Cynthia Gleason

Anthony Glenn

Alberto Gochez

Kaitlin Gold

Paolo Gonthier

Rena Gorovits

Erica Goss

Bruce Gossen

Sarah Green

Laura Grenville-Briggs

Rita Grosch

Lisa Grubisha

Niklaus Grünwald

David Guest

Abolfazl Hajihassani
Reza Hajimorad

Adam Hall

Jong Hyun Ham

Frédéric Hamelin

Richard Hamelin

John Hammond

Inge Hanssen

Sajeet Haridas

Dalphy Harteveld

Glen Hartman

John Hartung

Muhammad Nadeem Hassan

Michael Havey

Neil Havis

Nichola Hawkins

Rene Heim

Etienne Herrbach

Ulrich Hildebrandt

Mark Hilf

Bradley Hillman

Sarah Hind

Cory Hirsch

Iwai Hisashi

Ingerd Hofgaard

Monica Höfte

Sarah Holdgate

Cheng-Fang Hong

Jiahuai $\mathrm{Hu}$

Mengjun $\mathrm{Hu}$

Tzu-Pi Huang

Wenkun Huang

Alejandra Huerta

Teresa Hughes

Aminul Islam

Marie-Agnès Jaques

Michael Jeger

María del Mar

Jiménez-Gasco

Evan Johnson

Kenneth Johnson

Jeffrey Jones

Kathryn Jones

Richard Jones

Howard Judelson

Annemarie Justesen

Mehdi Kabbage

Zhian Kamvar

Prem Kandel

Yuba Kandel

Alexander Karasev

Matthew Kasson

Beat Keller

Sebastian Kiewnick

Eui-Joon Kil

Sun Tae Kim

Brian Knaus

Alyssa Koehler 
Jim Kolmer

Hideki Kondo

Olga Kozhar

Peter Kulakow

Sridhara Kunjeti

Di Kurtboke

Nemanja Kuzmanović

Tina Kyndt

Christophe Lacomme

Dilip Lakshman

Caixia Lan

Blanca Landa

Moshe Lapidot

Melen Leclerc

Alison Lees

Kirsten Leiss

Johan Leveau

Amit Levy

Julien Levy

Gina Lewin

Jinyun Li

Zhenghe Li

Judith Lichtezveig

Franz Lichtner

Hyoun-Sub Lim

Hannele Lindqvist-Kreuze

Kai-Shu Ling

Sébastien Lion

Ruey-Fen Liou

Christopher Little

Feng Liu

Junfeng Liu

Michele Loewen

Juan Jose Lopez-Moya

Emilia López-Solanilla

Camilo Lopez

Jennifer lorang

Mark Lubell

Chao-Xi Luo

Yong Luo

Wenxiu Ma

Laurence Madden

Walter Mahaffee

Anne-Katrin Mahlein

Kranthi Mandadi

Armelle Marais

Benoit Marçais

Rogerio Mardis

Camila Martini Zanella

Rick Masonbrink

Alex Mastin

Kerry Mauck

Dmitri Mavrodi

Brent McCallum

Greg McCollum

Mark McMullan

Neil McRoberts

Hillary Mehl

Lucky Mehra

Hajeewaka Mendis

Terence Mhora

Themis J. Michailides

Richard Michelmore

Timothy Miles

Alice Milne

Julia Minicka

Thomas Mitchell

Massimiliano Morelli
Izumi Mori

Enrique Moriones

Chris Mundt

Gary Munkvold

Jesus Murillo

Kenji Nakahara

Eric Newberry

Xianzhou Nie

Rients Niks

Jonathan Oliver

Richard Oliver

Marc Ongena

Peter Oudemans

Israel Pagán

Peter Palukaitis

Sheo Shankar Pandey

Zhiqian Pang

Hanu Pappu

Mathews Paret

Pierce Paul

Timothy Paulitz

Tamieka Pearce

Kerry Pedley

Hui Peng

Alvaro Perez-Quintero

Gregory Perry

Gary Peterson

Aparna Petkar

Tom Pizzolato

Randy Ploetz

Jesse Poland

Anissa Poleatewich

Sorina Popescu

Perrine Portier

Neha Potnis

Zacharias Pretorius

Claudia Probst

Dov Prusky

Jarosław Przetakiewicz

Alexander Putman

Pan Qinghua

Wenping Qiu

Feng Qu

Mahbubjon Rahmatov

Raman Rajagopal

Jeyaraman Rajaraman

Aurelie Rakotondrafara

Mandeep Randhawa

David Rasmussen

Massimo Reverberi

Camila Ribeiro

Jonathan Richards

Loup Rimbaud

Christophe Robaglia

Chris Rock

Marion Röder

Jeffrey Rollins

Cristina Rosa

Vittorio Rossi

Francesca Rotondo

Philippe Roumagnac

Matthew Rouse

Avijit Roy

Will Rutter

Jyoti Saini Sharma

Seiya Saito

Jonathon Sanchez

Helene Sanfacon
Surya Sapkota

Nobumitsu Sasaki

Leonardo Schena

Harald Scherm

Daniel Schlatter

Alexandra Schlenzig

Guido Schnabel

Torsten Schoeneberg

Herman Scholthof

Sebastian Schornack

Brenda Schroeder

Howard Schwartz

Francis Schwarze

Arne Schwelm

Ben Schwessinger

Erin Scully

Weixing Shan

Michael Shaw

Jason Shiller

Won-Bo Shim

Kenta Shirasawa

Maria Ines Siri

Ian Small

Theo Smits

Pilar Soengas

Jose Soriano

Adam Sparks

Rajagopalbab Srinivasan

Jeff Standish

Emil Stefańczyk

Andrei Steindorff

Arne Stensvand

Ioannis Stergiopoulos

Ann Stevens

Ethan Stewart

Jane Stewart

Lucy Stewart

Rajagopal Subramaniam

Karen Sullam

Leilani Sumabat

George Sundin

Nobuhiro Suzuki

Bryan Swingle

Les Szabo

Javier Tabima

Yu Takeuchi

Frank Takken

Firas Talas

Satyanarayana Tatineni

Andrew Taylor

Doron Teper

Shree Thapa

Marco Thines

Sara Thomas-Sharma

Gregory Thyssen

Chengming Tian

Sujan Timilsina

Sotirios E. Tjamos

Silvia Laura Toffolatti

Tania Toruno

Yukio Tosa

Hanh Tran

Eric Triplett

Lindsay Triplett

Pankaj Trivedi

Jane Trolinger

William Turechek

Ioannis Tzanetakis
William Underwood

Lisa Vaillancourt

Florence Val

Gary Vallad

Adrian Valli

Ariena van Bruggen

Sjaak van Heusden

Jan van Kan

Joel Vanneste

Arvind Varsani

Julio Vega Arreguin

Robert Venette

Ivana Vico

Sara Villani

Ron Walcott

Aiming Wang

Jie Wang

Nan-Yi Wang

Qi Wang

Todd Ward

Astri Wayadande

Melanie Weckert

Qingshan Wei

Alexandra Weisberg

Sean Westerveld

Andreas Westphal

David Wheeler

Terry Wheeler

Steve Whitham

Jonathan Whitworth

Emmanuel Wicker

Jean Williams-Woodward

Valerie Williamson

Laetitia Willocquet

Monika Wimmer

Ronja Wonneberger

Bo Ming Wu

Andy Wyenandt

Stephen Wyka

Stephen Wylie

Xianchun Xia

Ye Xia

$\mathrm{Jin} \mathrm{Xu}$

Xiangming $\mathrm{Xu}$

Guiping Yan

Qing Yan

Ching-Hong Yang

Wenwu Ye

Shyi-Dong Yeh

Karen Yin

Satoko Yoshida

Carolyn Young

Adam Zeilinger

Kurt Zeller

Quan Zeng

Murilo Zerbini

Jiasui Zhan

Baolong Zhang

Chuanqing Zhang

Jinfa Zhang

Yunzeng Zhang

Bingyu Zhao

LingXia Zhao

Shaobin Zhong

Wenqing Zhou 